\title{
Attitudes Towards Computerized CBT for Depression Amongst a Student Population
}

\author{
Nicky Mitchell \\ University of Portsmouth, UK \\ P. Kenneth Gordon \\ University of Southampton, UK
}

\begin{abstract}
Some studies of computerized cognitive behaviour therapy (CCBT) have found evidence of its effectiveness, yet a number have reported low uptake and/or completion rates. This study investigated attitudes towards CCBT for depression amongst 122 university students. The credibility of CCBT, expectancy-for-improvement and perceived likelihood of using it were all poor, although a minority $(9.8 \%)$ stated a preference for CCBT over other interventions. When 20 of the original sample received a demonstration of a CCBT programme for depression, significant increases in credibility, expectancy-for-improvement and in perceived likelihood of using CCBT were found. Numbers stating a preference for CCBT increased to $30 \%$. At both stages, most students stated a preference for CCBT to be accompanied by counselling. Qualitative analysis provided information about factors that might influence these attitudes. Implications for service delivery are discussed.
\end{abstract}

Keywords: Self-help, CCBT, depression, attitudes, credibility, expectancy.

\section{Introduction}

There is increasing interest in the use of self-help for emotional and mental health difficulties (Department of Health, 1999; Lewis et al., 2003). Lovell and Richards (2000) have argued the case for "stepped-care", in which less therapist-intensive, accessible treatments such as self-help are routinely offered first to many service users. Computerized cognitive behaviour therapy (CCBT) has been developed as an interactive self-help intervention that may include some non-specific features of the therapy relationship such as empathy, alliance and motivation (Cavanagh and Shapiro, 2004). CCBT programs exist for a variety of difficulties, and although the evidence-base remains small to date, certain programs are now recommended as one choice within the treatment of depression and anxiety (National Institute for Health and Clinical Excellence [NICE], 2006).

Therapists may still have reservations about CCBT; Whitfield and Williams' (2004) survey of BABCP-accredited therapists revealed a majority view of the approach as a supplement

Reprint requests to Nicky Mitchell, Counselling Service, Department for Curriculum and Quality Enhancement, Nuffield Centre, St Michael's Road, Portsmouth PO1 2ED, UK. E-mail: nicky.mitchell@ port.ac.uk 
rather than an alternative to clinician-guided therapy, with views that it would be less effective and lead to lower client satisfaction. A NICE-associated Health Technology Assessment report (Kaltenthaler et al., 2002; Kaltenthaler, Parry and Beverley, 2004) noted the lack of information about patient preferences, treatment acceptability and satisfaction in CCBT studies. It recommended qualitative studies of attitudes towards and experiences of CCBT. Little is known about the influences on service users' willingness to accept CCBT (Murray et al., 2003).

Information about attitudes towards CCBT may be suggested indirectly, for example from attrition figures. In a randomized controlled trial of "Beating the Blues" (BtB) (Proudfoot et al., 2003), 35\% of 89 program users ended prematurely. A BtB pilot by Van den Berg, Shapiro, Bickerstaffe and Cavanagh (2004) with 115 patients had a $45 \%$ attrition rate. Few users reportedly gave unfavourable feedback, but terminators were not followed up.

The tendency to use self-recruitment has made investigation of initial acceptability of CCBT difficult. However, in the Proudfoot et al. (2003) study, 32\% of 310 patients who were offered a screening appointment declined. Whitfield, Hinshelwood, Pashley, Campsie and Williams (2006) conducted a pilot of the CCBT program "Overcoming Depression", recruiting through clinical psychology referrals. Of 78 referrals offered a screening appointment, only $28 \%$ attended, although the authors suggested that this may be related to the secondary care setting and participants' anticipation of having clinician-guided treatment. Even when using selfreferrals, Marks et al. (2003) reported that $20 \%$ of 210 participants given a choice of four CCBT programs refused CCBT at screening stage.

Who then is likely to accept or decline CCBT? Murray et al. (2003) studied 81 patients who accepted or refused CCBT for bulimia. No differences were found in symptom severity, age, gender, or previous use/benefit of self-help, but non-engagers had significantly lower expectations of the usefulness of CCBT for themselves, although not for others. Misconceptions were reported about issues such as availability of therapist support. The authors stressed the need for patients' views about self-help to be explored and, where necessary, corrected, in order for its use to be better accepted.

Against this background, and the recommendations of NICE (2002), this study makes a preliminary exploration of attitudes to CCBT for depression amongst university students. The mental health needs of students have been noted (Royal College of Psychiatrists, 2003) as progressively approximating those of the general population, with increasing numbers seeking help for serious mental health problems. The attitudes under investigation are expectancy-forimprovement, credibility, perceived likelihood of use, intervention preference, and preference for therapist support. The first phase of the study investigates these attitudes at baseline, whilst the second tests whether a demonstration of the program, promoting socialization to CCBT (Beck, Rush, Shaw and Emery, 1979) can facilitate improved attitudes.

\section{Method}

\section{Participants and recruitment}

Participants were 122 students at Portsmouth University (61.5\% female, $36.9 \%$ male, 2 unknown, mean age 23.30), self-selected from a poster advertisement offering entry into a prize draw in return for helping the Counselling Service "with a questionnaire researching attitudes towards computerized self-help". Twenty participants from Stage One were randomly 
chosen for Stage Two from those expressing a willingness to participate further on consent forms. These were $75 \%$ female, $25 \%$ male, mean age 23.05 . They received a $£ 5$ music token for their time. As the gender balance of the university is $47.0 \%$ female, $53.0 \%$ male, these samples included a disproportionate number of female participants.

\section{Procedure}

Posters were placed in a number of public university locations. CCBT survey packs (detailed below and including information/consent forms), were left nearby. In Stage Two, participants attended the University Counselling Service individually, where the first author introduced a 30-minute demonstration of parts of the first two sessions of a CCBT program by telling participants that they were to have a brief orientation to the program without actually participating in it, in order to give them a live experience of the range of formats used within the program, and to get an overall "feel" for it. They were informed that the interviewer would complete selected parts of the program as might a mildly depressed user. Attitude scales (Borkovec and Matthews, 1988) were repeated immediately after this.

\section{Materials}

Demographics form. This included questions about age, gender, and experiences/ treatment of anxiety/depression.

Credibility/expectancy scales (Borkovec and Matthews, 1988). These scales were designed to test for equitable expectancy and rationale credibility in controlled clinical trials including CBT (Borkovec and Costello, 1993; Borkovec, Newman, Pincus and Lytle, 2002). They consist of 9-point Likert-type ratings of three aspects of credibility (treatment logic, expected success, and confidence in recommending to a friend). Expectancy-for-improvement is rated as a percentage of how much improvement the participant thinks will occur from the intervention.

Additional questions. The credibility/expectancy scales were extended for this study to include a percentage rating of the perceived likelihood of using four interventions (counselling, self-help book, CCBT and Internet) if depressed. Participants also specified their first-choice intervention (from these four, or an "other" category). Finally, they were asked whether they would prefer to use CCBT with or without also speaking to a counsellor. Open questions were used to elicit information on reasons for preferences, and perceived advantages/disadvantages of CCBT (from Murray et al., 2003). The full questionnaire (available from the authors) includes detailed instructions, intended to ensure that respondents answer the items as if they are currently experiencing depression.

"Overcoming Depression" CD-ROM (Williams, Taylor, Aubin, Harkin and Cottrell, 2002). This six-session interactive CD-ROM uses CBT principles to treat adults with mild to moderate depression. Although this program has an insufficient evidence-base to date for recommendation by NICE (2006), it was used in this study simply for demonstration purposes and, as such, appears to offer a valid example of current practice. 


\section{Analysis}

The data were analysed using SPSS-11 for Windows. Given that most of the questionnaires involved ordinal ratings, a non-parametric approach was chosen. Since the participants were predominantly female, the Mann-Whitney test was used to assess any effects of gender. There were no significant differences for any of the study variables. Raw data are presented on initial attitudes, and changes between Stages One and Two are assessed using the Wilcoxon Signed Ranks test (two-tailed). A simple qualitative analysis, based on the method of Boyzatis (1998), was used to identify themes within the responses to open questions. Thematic codes were initially assigned by the first investigator and then subjected to a reliability check, in which the second investigator independently coded responses from a random sample of 50 Stage One participants. Inter-rater agreement was satisfactory, ranging from $81.0 \%$ to $87.3 \%$ across questions, so no changes were made to the codings.

\section{Results}

Stage One

Mental health information. Eighty participants (65.6\%) identified themselves as having previous or current difficulties with either depression or anxiety (more specific detail was not requested); a further $9.8 \%$ indicated uncertainty about this. Counselling had been used by $40.2 \%, 28.7 \%$ had read a self-help book, $77.9 \%$ had used the Internet for health reasons, and $0.8 \%$ had used CCBT.

\section{Attitudes towards CCBT for depression}

Credibility. Total scores on the three items ranged between 3 and 26 (maximum credibility $=27)$, with mean $(M)=12.20$ and standard deviation $(S D)=5.25$.

Expectancy-for-improvement. Scores ranged between $0 \%$ and $70 \%, M=34.7 \%(S D=$ $19.4 \%)$.

Likelihood of using each intervention. The mean perceived likelihood of each targeted intervention being used if depressed was: counselling 61.8\% $(S D=26.6 \%)$, Internet $60.9 \%$ $(S D=27.8 \%)$, CCBT $47.6 \%(S D=29.7 \%)$, self-help book $43.0 \%(S D=26.9 \%)$.

Intervention preference. Counselling was the preferred choice of $54.9 \%$ of participants, followed by the Internet (17.2\%), CCBT (9.8\%), self-help book $(6.6 \%)$ and other interventions (7.4\%). A variety of reasons were cited for choices, with typical themes being the interpersonal nature of counselling, versus the privacy and convenience of self-help methods. Full details are given in Table 1.

Advantages and disadvantages of using CCBT. A more detailed picture emerges from the thematic analysis of data on these questions, with similar interpersonal and practical factors emerging, as shown in Table 2.

Preference for using CCBT for depression with or without additional counselling. $73.9 \%$ of participants stated a preference, if using CCBT, to do so alongside counselling, while $26.1 \%$ 
Table 1. Thematic analysis of intervention preferences (Stage One -122 participants)

\begin{tabular}{|c|c|c|c|c|}
\hline $\begin{array}{l}\text { Counselling } \\
(54.9 \%)\end{array}$ & Internet $(17.2 \%)$ & CCBT $(9.8 \%)$ & $\begin{array}{l}\text { Self-help book } \\
\quad(6.6 \%)\end{array}$ & $\begin{array}{l}\text { Other } \\
(7.4 \%)\end{array}$ \\
\hline Better to talk (35) & Privacy (16) & Privacy (10) & Privacy (4) & Friends (5) \\
\hline $\begin{array}{l}\text { Interpersonal } \\
\text { factors }(26)\end{array}$ & $\begin{array}{l}\text { Accessibility (12) } \\
\text { Cost (3) }\end{array}$ & $\begin{array}{l}\text { Independent self-help (3) } \\
\text { Initial self-management (3) }\end{array}$ & Accessibility (2) & $\begin{array}{l}\text { Family (5) } \\
\text { Medical (2) }\end{array}$ \\
\hline $\begin{array}{l}\text { Tailored } \\
\text { treatment (13) }\end{array}$ & & Interactivity (2) & & \\
\hline $\begin{array}{l}\text { Previous } \\
\quad \text { experience (6) }\end{array}$ & & & & \\
\hline
\end{tabular}

Tabulated data refer to the frequency of each intervention being cited as first choice (excluding $4.1 \%$ missing data), and the breakdown of reasons given (number citing each theme in brackets).

Table 2. Perceived advantages and disadvantages of using CCBT if depressed (Stage One)

\begin{tabular}{ll}
\hline Advantages & \multicolumn{1}{c}{ Disadvantages } \\
\hline Privacy (53) & Impersonal (39) \\
Accessibility (43) & Limited/general ideas (23) \\
Info/techniques/advice (8) & Symptom-related (12) \\
First step (7) & Isolation (11) \\
Cost (6) & Talking helps (10) \\
"None" (5) & Commitment problems (9) \\
Diagnosis/problem recognition (4) & Computer issues (8) \\
Non-judgement (4) & No monitoring/clarification (5) \\
Opportunity to help self (4) & Less confidence in approach (4) \\
Distraction from depression (3) & Could feel worse (4) \\
Familiarity of technology (3) & Possible misinformation (3) \\
& "None" (2) \\
& Cost (2) \\
& Cynicism (2) \\
& Feel “palmed off" (2) \\
& Boring (2) \\
\hline
\end{tabular}

Note: The number of participants citing each theme is shown in brackets.

preferred to use CCBT alone. Of participants who indicated CCBT as their first choice, only $25 \%$ preferred to have additional counselling.

\section{Stage Two}

Where before/after changes are reported below, the "before" data refer to the sub-sample who took part in stage two, not the whole group. Effect size was calculated to illustrate the magnitude of change, using Cohen's benchmark of $r \geqslant 0.5$ to represent a large effect (Cohen, 1988, cited by Field, 2005). As shown, data exceed this benchmark for credibility, expectancy-for-improvement, and likelihood of using CCBT for depression. 
Credibility. Total scores ranged between 8 and 25 after the demonstration $(M=19.20$, $S D=4.79)$. There was a significant increase in credibility with the median increasing from 13.50 to $20.50(z=-3.29, p<0.001, r=-0.52)$.

Expectancy-for-improvement. Scores ranged between $30 \%$ and $80 \%, M=60.0 \%$ (SD= $15.6 \%)$. There was a significant increase in expectancy scores after the demonstration, with the median increasing from $40.0 \%$ to $60.0 \%(z=-3.54, p<.001, r=-0.56)$.

Likelihood of using each intervention. Mean likelihood of using CCBT was $69.5 \%$ $(S D=24.2 \%)$. After the demonstration, there was a significant increase in likelihood of CCBT being chosen, with the median increasing from $40.0 \%$ to $80.0 \%$ ( $z=-3.22, p=.001$, $r=-0.51)$. There were no significant changes for any other intervention choice.

Intervention preference. Choice of CCBT as the preferred intervention increased from three $(15 \%)$ to six $(30 \%)$ in Stage Two, with a corresponding decrease in preference for counselling. Sample size prevented statistical analysis of the significance of this change.

Perceived advantages/disadvantages of using CCBT if depressed. The themes that emerged overlapped with those elicited in Stage One, with the privacy of CCBT being most commonly cited as an advantage ( $40 \%$ of participants). A number of comments also suggested a positive shift in attitude as a result of the demonstration. Examples include: "I expected it to be like a book and was surprised it had audio and video clips", "more interactive than I expected", "I no longer worry that it isn't personal enough". Other positive comments include: "very informative, asks you questions which it then relates into your own circumstances", "appears to understand many different problems, and suggests realistic targets", "non-critical, non-threatening", and "motivating". Where disadvantages were given, these echoed previous themes (CCBT lacking specificity, being impersonal). Comments that seemed to relate specifically to viewing the programme included the following: "a lot of work, may feel unachievable", "perhaps more feedback should be included", and "long winded could be hard to concentrate for long".

Preference for using CCBT for depression with or without additional counselling. Fifteen (75\%) of Stage Two participants stated a preference for using CCBT in addition to counselling, with five $(25 \%)$ preferring to use CCBT alone.

\section{Discussion}

\section{Pre-demonstration attitudes towards CCBT}

In Stage One, students' attitudes towards CCBT following only a brief description of the approach were examined. Participants were not convinced of the credibility of CCBT for depression at this stage. Credibility ratings produced a per-item mean of 4.07, which was below the mid-point on the nine-point scale, suggesting CCBT was seen as less than "somewhat credible". By comparison, Borkovec and Costello (1993) and Borkovec et al. (2002) reported credibility figures of 6.95 and 7.58 respectively for therapist-guided CBT (TCBT). The mean expectancy score of $34.7 \%$ is substantially lower than the $68.5 \%$ and $67.5 \%$ reported in these two studies, and suggests that participants had moderately low expectancy-for-improvement from CCBT. The perceived likelihood of using CCBT, at $47.6 \%$, is lower than that for counselling or the Internet. However, the finding that almost $10 \%$ of participants rated CCBT as 
their first choice intervention suggests that a minority of participants may be actively interested in such an intervention.

Participants citing counselling as their first choice reported their reasons as including the importance of talking and interpersonal factors. Privacy and treatment accessibility appeared to be important considerations for participants stating a preference for any of the self-help options, and also for participants considering the advantages of CCBT (mentioned by $43.4 \%$ and $35.2 \%$ of participants respectively). At the same time, CCBT was cited as impersonal by one-third of participants, and as likely to provide only limited or general ideas. Schmidt (2003) has discussed evidence contradicting the view of CCBT as impersonal, and some of the disadvantages cited in this study may be based on misconceptions (Murray et al., 2003). Whether based on reality or misconception however, such factors may be relevant to an individual's treatment preferences.

\section{Impact of CCBT demonstration}

The hypothesis that a CCBT demonstration would impact on attitudes towards CCBT was confirmed. Specifically, after demonstration, scores for credibility, expectancy-forimprovement and likelihood of using CCBT all rose by at least 50\%, and despite the small sample size, the increases were highly significant, with large effect sizes. CCBT was cited as the preferred intervention twice as often at this stage, albeit with an inadequate sample size for statistical analysis. In terms of clinical impact, the per-item mean credibility of 6.40 represents at least a "somewhat credible" label, although it remains lower than TCBT figures reported above. The mean expectancy rises to $60 \%$, but again it remains lower than TCBT figures. The $69.5 \%$ likelihood of using CCBT is higher than the mean likelihood of attending counselling cited by participants in Stage One and appears "moderately high". These changes were reflected in subjective comments, with more favourable views towards CCBT emerging. Some comments suggest the reduction of misconceptions. Positive attitudes were not universal, however, and some participants remained unlikely to choose to use CCBT.

\section{Preference for using CCBT with or without counselling}

A large majority of participants preferred the idea of CCBT alongside therapeutic support both prior to and after the CCBT demonstration. This might support the NICE (2002) recommendation of ongoing monitoring of CCBT users, although this study does not determine whether participants had in mind monitoring or therapy. There is some preliminary indication in the study that the participants who rated CCBT as their first choice were particularly likely to prefer CCBT without additional input. These are students that CCBT might very usefully target.

\section{Study limitations}

Caution is needed in generalizing from these data to a wider population. Whilst students' mental health needs increasingly match those of the wider population, there are differences in this group, notably in educational achievement, but probably also in particular attitudes. It seems reasonable to hypothesize that students may be more likely to have favourable attitudes towards technology than the general public. If this is true, the rather low confidence in CCBT 
may be larger in other populations. Additionally, a surprisingly large proportion of the sample reported personal experience of depression or anxiety. This could mean that people with current depressed mood had tended to volunteer for the survey. If so, they would be more representative of a real life situation in which CCBT was offered. It could be that attitudes towards CCBT would be more positive for those who are depressed (as found in relation to counselling by Halgin, Weaver, Edell and Spencer, 1987), although as these authors also found that previous input leads to intervention allegiance, the large number (40.2\%) who had accessed counselling may instead have skewed the data towards a preference for face-to-face contact. Alternatively, respondents may have simply used the terms depression and anxiety to refer to temporary mood states, rather than mental health disorders. More exact information on current mental health status and a wider participant pool would be useful in any future study.

The short demonstration with a clinician present is a rather different experience from sitting alone for a prolonged period when depressed attempting to use a CCBT program. It may be more similar to the therapist-guided CCBT that appeared to be preferred by the majority in this study. An opportunity for participants to simply familiarize themselves with the package could be another option worth testing.

The qualitative analysis provides a flavour of participants' attitudes towards CCBT, but must be interpreted cautiously. Whilst the study attempted to avoid generalization by clearly asking for any advantages "for you" of using CCBT, not all participants may have acknowledged this. It cannot be ruled out that, as with Murray et al.'s (2003) findings with bulimic clients, CCBT was seen by some as having advantages for others but not themselves. Finally, identification of advantages/disadvantages may reveal little about actual uptake of CCBT, as found by Murray et al. (2003). An interview methodology might offer one way to gain more clarification and elaboration of themes emerging here.

\section{Implications for clinical practice}

Attitudes towards CCBT found in Stage One may provide some insight into the low uptake reported in some studies. Only one participant had previously used CCBT and given its relative novelty it may be unrealistic to expect very favourable attitudes at this time. Such attitudes need to be facilitated if the numbers of individuals who may benefit from such treatment are to be reached. This study suggests that a simple demonstration, perhaps offered as a "taster session", may be an effective way to do so within services where CCBT is used.

A further potential obstacle to CCBT acceptance may relate to methods of referral. When individuals are referred or refer themselves to psychological therapy, they may expect an interpersonal treatment, which could reduce the appeal and acceptability of alternative interventions. It is possible that CCBT offered as easily accessed self-help, before a therapy referral, may improve uptake (Whitfield et al., 2006). This is borne out by our participants' endorsement of CCBT accessibility and its role as a first step.

A small number of participants in this study cited a requirement to attend a clinic to use CCBT as a disadvantage, and accessibility was a frequently cited advantage of all the selfhelp options. The development of online versions of CCBT, available for use at home, may therefore increase its acceptability.

In conclusion, this study suggests that, if CCBT is to be used to rectify the shortfall of CBT provision for mild to moderate depression, services must address user attitudes and preferences. 
Socialization to the approach seems vital, using methods such as the demonstration of materials adopted here. CCBT must not be wholly separated from therapist contact if it is to be acceptable to larger numbers of service users. CCBT may never be the first choice option for a majority; however, it may serve the needs of a group who might not otherwise be effectively helped, and it might have a place in both Primary Care and Higher Education as a first line treatment for depressed individuals.

\section{References}

Beck, A. T., Rush, A. J., Shaw, B. F. and Emery, G. (1979). Cognitive Therapy of Depression. New York: Guilford.

Borkovec, T. D. and Mathews, A. M. (1988). Treatment of nonphobic anxiety disorders: a comparison of nondirective, cognitive, and coping desensitization therapy. Journal of Consulting and Clinical Psychology, 56, 877-884.

Borkovec, T. D. and Costello, E. (1993). Efficacy of applied relaxation and cognitive-behavioral therapy in the treatment of generalized anxiety disorder. Journal of Consulting and Clinical Psychology, 61, 611-619.

Borkovec, T. D., Newman, M. G., Pincus, A. L. and Lytle, R. (2002). A component analysis of cognitive behavioral therapy for generalized anxiety disorder and the role of interpersonal problems. Journal of Consulting and Clinical Psychology, 70, 288-298.

Boyzatis, R. E. (1998). Transforming Qualitative Information: thematic analysis and code development. California: Sage.

Cavanagh, K. and Shapiro, D. A. (2004). Computer treatment for common mental health problems. Journal of Clinical Psychology, 60, 239-251.

Cohen, J. (1988). Statistical Power Analysis for the Behavioural Sciences (2nd Ed.). New York: Lawrence Erlbaum.

Department of Health (1999). A National Service Framework for Mental Health. London: Department of Health.

Field, A. (2005). Discovering Statistics Using SPSS (2nd Ed.). London: Sage.

Halgin, R. P., Weaver, D. D., Edell, W. S. and Spencer, P. G. (1987). Relation of depression and helpseeking history to attitudes toward seeking professional psychological help. Journal of Counseling Psychology, 34, 177-185.

Kaltenthaler, E., Parry, G. and Beverley, C. (2004). Computerized cognitive behaviour therapy: a systematic review. Behavioural and Cognitive Psychotherapy, 32, 31-55.

Kaltenthaler, E., Shackley, P., Stevens, K., Beverley, C., Parry, G. and Chilcott, J. (2002). A systematic review and economic evaluation of computerized cognitive behaviour therapy for depression and anxiety: executive summary. Health Technology Assessment, 6, 22.

Lewis, G., Anderson, L., Araya, R., Elgie, R., Harrison, G., Proudfoot, J., Schmidt, U., Sharp, D., Weightman, A. and Williams, C. (2003). Self-Help Interventions for Mental Health Problems. Report to the Department of Health R and D Programme. http://www.nimhe.org.uk/downloads/self-help.pdf

Lovell, K. and Richards, D. (2000). Multiple access points and levels of entry (MAPLE): ensuring choice, accessibility and equity of CBT services. Behavioural and Cognitive Psychotherapy, 28, 379-391.

Marks, I. M., Mataix-Cols, D., Kenwright, M., Cameron, R., Hirsch, S. and Gega, L. (2003). Pragmatic evaluation of computer-aided self-help for anxiety and depression. British Journal of Psychiatry, 183, 57-65.

Murray, K., Pombo-Carril, M. G., Bara-Carril, N., Grover, M., Reid, Y., Langham, C., Birchall, H., Williams, C., Treasure, J. and Schmidt, U. (2003). Factors determining uptake of a CD-ROM-based 
CBT self-help treatment for bulimia: patient characteristics and subjective appraisals of self-help treatment. European Eating Disorders Review, 11, 243-260.

National Institute for Clinical Excellence (NICE) (2002). Guidance on the use of computerised cognitive behavioural therapy for anxiety and depression. Technology Appraisal Guidance No. 51 . London: NICE.

National Institute for Health and Clinical Excellence (NICE) (2006). Computerised Cognitive Behaviour Therapy for Depression and Anxiety: review of technology appraisal 51. NICE Technology Appraisal 97. London: NICE.

Proudfoot, J., Goldberg, D., Mann, A., Everitt, B., Marks, I. and Gray, J. A. (2003). Computerized, interactive, multimedia cognitive-behavioural program for anxiety and depression in general practice. Psychological Medicine, 33, 217-227.

Royal College of Psychiatrists (2003). The Mental Health of Students in Higher Education. Council Report CR112. http://www.rcpsych.ac.uk/publications/cr/council/cr112.pdf

Schmidt, U. (2003). Getting technical. European Eating Disorders Review, 11, 147-154.

Van den Berg, S., Shapiro, D. A., Bickerstaffe, D. and Cavanagh, K. (2004). Computerized cognitivebehaviour therapy for anxiety and depression: a practical solution to the shortage of trained therapists. Journal of Psychiatric and Mental Health Nursing, 11, 508-513.

Whitfield, G. and Williams, C. (2004). If the evidence is so good, why doesn't anyone use them? A national survey of the use of computerized cognitive behaviour therapy. Behavioural and Cognitive Psychotherapy, 32, 57-65.

Whitfield, G., Hinshelwood, R., Pashley, A., Campsie, L. and Williams, C. (2006). The impact of a novel computerized CBT CD Rom (Overcoming Depression) offered to patients referred to clinical psychology. Behavioural and Cognitive Psychotherapy, 34, 1-11.

Williams, C. J., Taylor, L., Aubin, S. D., Harkin, P. J. R. and Cottrell, D. (2002). Overcoming Depression: a five areas approach self-help package. Leeds: Calipso/University of Leeds (www.calipso.co.uk). 